\title{
Clinical profile of pathological jaundice among neonates admitted in the National Referral Hospital, Bhutan
}

\author{
Kinzang Dechen ${ }^{1}$, Tenzin Lhadon², Mimi Lhamu Mynak³, Phurpa ${ }^{4}$ \\ ${ }^{1,4}$ Faculty of Postgraduate Medicine, Khesar Gyalpo University of Medical Sciences of Bhutan, Thimphu, Bhutan \\ ${ }^{2,3}$ Department of Pediatrics, Jigme Dorji Wangchuck National Referral Hospital, Thimphu, Bhutan
}

\begin{abstract}
Introduction: Neonatal jaundice is a common condition. There are various maternal and neonatal clinical characteristics that have been described regarding pathological jaundice. We aimed to describe the clinical profile of pathological jaundice and to estimate its prevalence among newborns admitted at the National Referral Hospital of Bhutan. Methods: A descriptive study design was used to study cases of pathological jaundice sadmitted at the Gyaltsuen Jetsun Pema Neonatal Intensive Care Unit (NICU) of the National Referral Hospital of Bhutan, for a period of 1 year from $7^{\text {th }}$ November 2018 to $6^{\text {th }}$ November 2019. Data was collected using a predesigned case proforma, entered and analyzed in EpiData after obtaining ethical clearance from the Research Ethics Board of Health, Bhutan. Descriptive analysis was done for the socio-economic characteristics. Chi square test was used to find the significant association between categorical variables at 5\% significant level. Results: ABO blood group incompatibility and neonates less than one week of age were found to be most common clinical profile. The neonates born to primiparous mothers were 1.45 times $(95 \%$ CI $1.07-2.00)$ more likely to suffer from excessive weight loss compared with multiparous mothers and it this association was highly significant ( $p$-value of 0.016 ). Facility based prevalence of pathological neonatal jaundice was $63.66 \%$. The median age on admission was 4 days. Conclusions: ABO incompatibility, neonates less than one week of age, primiparous mothers with feeding issues should be closely followed or screened for pathological jaundice especially during the first one week of life. The prevalence of pathological jaundice was observed to be high in our setting.
\end{abstract}

Keywords: Blood group incompatibility; Feeding issues; Pathological jaundice; Prevalence; weight loss.

\section{INTRODUCTION}

Neonatal jaundice is a very common condition worldwide occurring in up to $60 \%$ of term and $80 \%$ of preterm newborns ${ }^{1}$. It is one of the main reasons for neonatal admissions and morbidity especially during the first week of life.

Jaundice in newborns can be physiological or pathological. Jaundice attributable to physiological immaturity of neonates to handle increased bilirubin production is termed as physiological jaundice ${ }^{2}$. The elevated bilirubin in newborns is due to the immature hepatic metabolism of bilirubin, the increased red blood cell turnover, and the shorter lifespan of red blood cells.

Pathological jaundice is defined as any newborns requiring either phototherapy or exchange transfusion for the

Corresponding author:

Kinzang Dechen

k.dechen1990@gmail.com treatment of jaundice ${ }^{2}$. Severe neonatal jaundice is defined as any pathological jaundice whose Total Serum Bilirubin (TSB) level falls above the exchange transfusion range ${ }^{3}$. The TSB level depends upon the age and gestation age of the baby.

Neonatal jaundice may not be a major cause of mortality but is still an important cause of morbidity especially in developing countries. Severe neonatal jaundice can lead to serious and irreversible neurodevelopment consequences if not treated timely.

Studies around the world have described various clinical profile and incidence of severe neonatal jaundice. The incidence of severe neonatal jaundice was found to be highest in low to middle income countries like in Southeast Asian region. The lowest incidence was found in more developed regions ${ }^{3,4}$. In 2016, neonatal death due to neonatal jaundice was ranked seventh globally among all causes of neonatal deaths ${ }^{4}$.

There are many known maternal and neonatal risk factors that cause pathological jaundice ${ }^{1,2}$. Neonatal factor includes neonates in their first week of life, prematurity, low birth weight, male child, inadequate feeding or excessive weight loss, $\mathrm{ABO}$ and Rh incompatibility, birth asphyxia, cephalohematoma, 
sepsis and previous siblings with jaundice requiring treatment. Maternal factors like primiparity, maternal age, prolonged labour, use of oxytocin, and maternal co morbidities are also known to cause jaundice. Apgar score was used to assess the condition of the baby soon after birth at 1 and 5 minutes.

Since no study has been conducted on neonatal jaundice in our setting this research was designed to study the clinical profiles of pathological jaundice. This study provides a baseline data on clinical profile and prevalence of pathological jaundice in the National Referral Hospital.

\section{METHODS}

After obtaining ethical clearance from the Research Ethics Board of Health, Ministry of Health, Bhutan vide letter number Ref. No. REBH/Approval/2018/060 dated $2^{\text {nd }}$ October 2018, a descriptive study conducted at the Gyaltsuen Jetsun Pema Neonatal Intensive Care Unit (NICU) of Jigme Dorji Wangchuck National Referral Hospital (JDWNRH) from $7^{\text {th }}$ November 2018 to $6^{\text {th }}$ November, 2019. JDWNRH serves as the National Referral hospital in Bhutan and NICU includes the critical care unit with 14 beds and the nursery unit with 23 beds.

There were 2493 neonates admitted in the NICU of which 1587 had pathological jaundice. Of 1587 neonates with pathological jaundice, 407 neonates were recruited for the study using systematic random sampling. as shown in the Figure below.

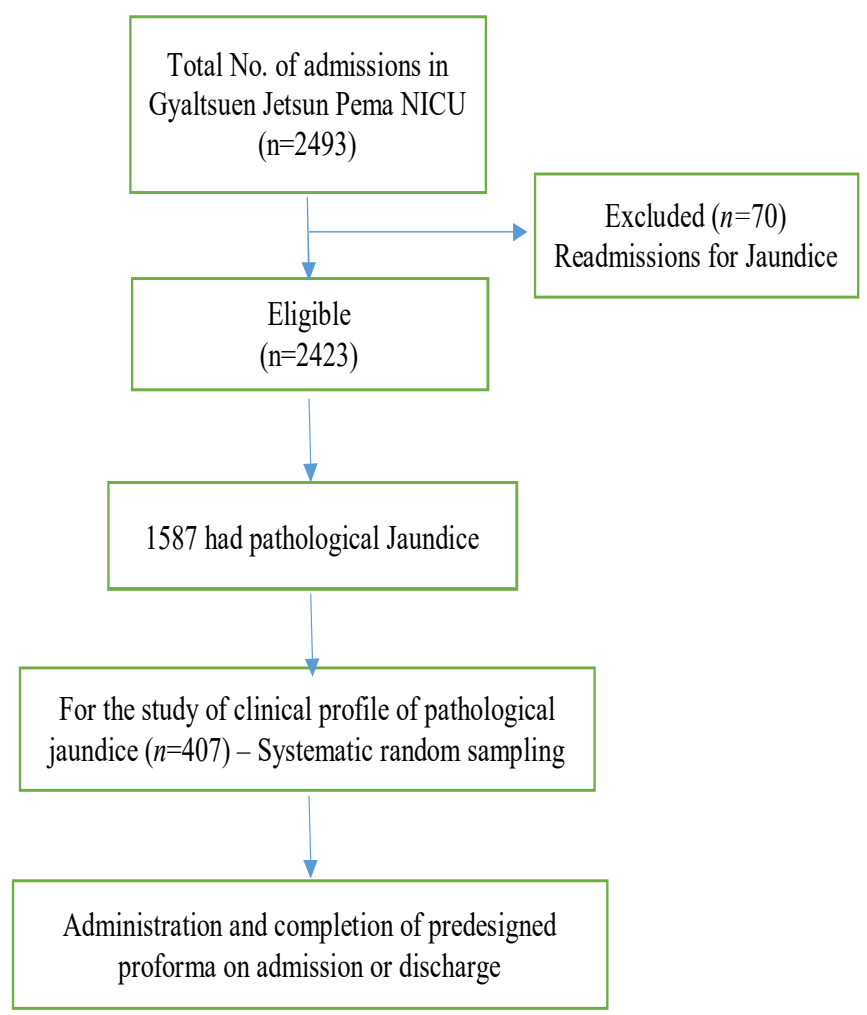

Figure 1. Flowchart of patient recruitment and data collection
Data collection was done using a self-developed case proforma after reviewing the existing literature which was pilot tested and validated by the experts in the field. Demographic details, clinical history, examination and investigation findings were collected using the proforma and from the Maternal and Child Health handbook. Neonatal characteristics included were age, sex, gestation age, birth weight, $\mathrm{ABO}$ and $\mathrm{Rh}$ incompatibility, sepsis, cephalohematoma, excessive weight loss and feeding issues. Maternal characteristics included were age and parity of mothers, use of oxytocin for labour, prolonged labour and comorbidities like Gestational Diabetes Mellitus (GDM), gestational hypertension and thyroid disorders.

Data collected was entered on a password protected computer while the hard copies of the proforma were stored in a secured location. No personal identifiers of the informants were used. Data were checked for completeness and double entered into Epidata version 3.1 to eliminate errors in data entry. Data management, processing and analysis were done using Epidata Analysis version V2.2.2.183.

Variables were coded and descriptive analysis were performed. Demographic and clinical characteristics were given in frequencies with their percentages. Median was calculated to determine the age on presentation of neonates with jaundice. Graphical methods and tables were also used to summarize the descriptive statistics. Fisher exact test was used to assess the association between parity and excessive weight loss.

Prevalence of pathological jaundice was calculated by dividing the total number of neonates with pathological jaundice by the total number of neonates admitted in NICU of JDWNRH during the one year study period.

\section{RESULTS}

During the study period, 2493 neonates were admitted at the Gyaltsuen Jetsun Pema NICU, JDWNRH. Out of the total admissions, 344 were admitted in critical care unit and the remaining 2149 neonates in the nursery unit. Of the total admissions, 1587 (63.66\%) babies had pathological jaundice.

\section{Neonatal Characteristics \\ Clinical presentation}

In our study, of 407 neonates recruited, $86.98 \%$ of them had icterus till lower legs/arms and palms and soles.

On admission, $30(7.37 \%)$ neonates had fever, 145 (35.63\%) had feeding issues, 5 (1.23\%) had cephalohematoma and none had features of neurological dysfunction.

From 407 babies, $360(88.45 \%)$ were born at term and $47(11.55 \%)$ were born preterm. Out of these neonates, 354 babies $(85.01 \%)$ had birth weight of more than 2500 grams and $10(2.46 \%)$ had very low birth weight of less than 1500 grams. The study found that $78 \%$ of the neonates developed jaundice within their first week of life. The median age on the diagnosis of jaundice was 4 days. 
Table 1. Birth characteristics of neonates with pathological jaundice admitted in $\mathrm{NICU}^{*}$ of $\mathrm{JDWNRH}^{\dagger}$ from 2018 $2019(n=407)$

\begin{tabular}{ll}
\hline Birth characteristics & $\boldsymbol{n}(\boldsymbol{\%})$ \\
\hline $\begin{array}{l}\text { Type of birth } \\
\text { Term birth }\end{array}$ & $360(88.45)$ \\
Preterm birth & $47(11.55)$ \\
Sex & \\
Male & $246(60.44)$ \\
Female & $161(39.56)$ \\
Birth weight ( in grams) & \\
$\leq 1500$ & $10(2.46)$ \\
$1501-2499$ & $51(12.53)$ \\
$2500-3999$ & $334(82.06)$ \\
$\geq 4000$ & $12(2.95)$ \\
Apgar at 5 minutes & \\
Less than 7 & $2(0.49)$ \\
7 or more & $405(99.51)$ \\
\hline
\end{tabular}

${ }^{*}$ Neonatal Intensive Care Unit, ${ }^{\dagger}$ Jigme DorjiWangchuck National Referral Hospital

Table 2. Clinical characteristics of neonates with pathological jaundice admitted in NICU*, JDWNRH', 2018-2019 $(n=407)$

\begin{tabular}{ll}
\hline Clinical characteristics & $\boldsymbol{n}(\mathbf{\%})$ \\
\hline ABO $^{\ddagger}$ or $\mathbf{R h}^{\S}$ incompatibility & \\
$\mathrm{ABO}$ incompatibility & $0(22.11)$ \\
$\mathrm{Rh}^{\S}$ incompatibility & $317(77.89)$ \\
$\mathrm{No}^{\mathrm{ABO}} / \mathrm{Rh}^{\S}$ incompatibility & \\
Excessive weight loss & $122(29.97)$ \\
Yes & $285(70,03)$ \\
No & \\
Feeding issues & $145(35.63)$ \\
Yes & $262(64.37)$ \\
No & \\
Neonatal sepsis & $24(5.90)$ \\
Yes & $383(94.10)$ \\
No & \\
Cephalhematoma & $5(1.23)$ \\
Yes & $402(98.77)$ \\
No & \\
\hline
\end{tabular}

${ }^{*}$ Neonatal Intensive Care Unit, ${ }^{\dagger}$ Jigme Dorji Wangchuck National Referral Hospital, ${ }^{\ddagger}$ Blood Group, ${ }^{\S}$ Rhesus factor, "Excessive weight loss - Loss of $10 \%$ or more of birth weight
Ninety (22.11\%) neonates had ABO incompatibility. None had $\mathrm{Rh}$ incompatibility nor isoimmune hemolytic disease. One hundred forty five (35.63\%) newborns had feeding issues like inadequate breast milk production, poor feeding technique, etc. Excessive weight loss was observed in 122 (29.98\%) neonates.

Sixty-one (31.44\%) neonates of the 194 multiparous mothers had a history of previous siblings or family history of neonatal jaundice requiring treatment. However, out of these neonates, 16 had ABO incompatibility, 15 had feeding issues and 13 were male babies.

There were 23 cases of severe pathological jaundice but none required exchange transfusion. Neonates with $\mathrm{ABO}$ incompatibility $^{13}$ and feeding issues ${ }^{7}$ were found to be the most common clinical profile.

Table 3. Association between parity and excessive weight loss in neonates with pathological jaundice in NICU*, JDWNRH ${ }^{\dagger}$, 2018-2019 $(n=407)$

\begin{tabular}{|c|c|c|c|c|}
\hline Parity & $\begin{array}{l}\text { Number of } \\
\text { neonates } \\
\text { with exces- } \\
\text { sive weight } \\
\text { loss } \\
(n=122)\end{array}$ & $\begin{array}{l}\text { Number of } \\
\text { neonates } \\
\text { with no } \\
\text { excessive } \\
\text { weight loss } \\
(n=285)\end{array}$ & $95 \% \mathrm{CI}^{*}$ & $p$-value \\
\hline Primi $^{\S}$ & 75 & 138 & \multirow{2}{*}{$\begin{array}{l}1.45 \\
(1.07- \\
2.00)\end{array}$} & \multirow[t]{2}{*}{0.016} \\
\hline Multi" & 47 & 147 & & \\
\hline
\end{tabular}

${ }^{*}$ Neonatal Intensive Care Unit, ${ }^{\dagger}$ Jigme DorjiWangchuck National Referral Hospital, ${ }^{*}$ Confidence Interval, ${ }^{\S}$ Primiparous, "Multiparous

\section{Maternal Characteristics}

Among the 407 mothers included, 317 (77.88\%) mothers were between 21 and 34 years of age, $31(7.62 \%)$ were teenagers and remaining $59(14.50 \%)$ were 35 years of age and above.

Two hundred and thirteen $(52.33 \%)$ mothers were primiparous and the remaining 194(47.67\%) mothers were multiparous. In our study, $40 \%$ of primiparous mothers had some sort of feeding issues among which $35 \%$ of their neonates had excessive weight loss. Among the multiparous mothers, $30 \%$ of them had feeding issues and $25 \%$ newborns had excessive weight loss. The neonates born to primiparous mothers were 1.45 times (95\% CI $1.07-2.00)$ more likely to suffer from excessive weight loss compared with multiparous mothers and it this association was highly significant ( $p$-value of 0.016 ) 
Table 4. Demographic and clinical characteristics of mothers of neonates with pathological jaundice admitted at NICU*, JDWNRH ${ }^{\dagger}$, 2018-2019 $(n=407$

\begin{tabular}{lll} 
Variables & $n$ & $(\%)$ \\
\hline
\end{tabular}

Age (in years)

$\leq 20$

31

7.62

21-34

317

77.88

$\geq 35$

59

14.50

Parity

Primiparous

213

Multiparous

194

Gestation age (in weeks)

$<37$

47

11.55

$\geq 37$

360

88.45

Oxytocin induction

Yes

109

26.78

No

298

73.22

Prolonged labour

Yes 13

No

394

96.81

Gestational Hypertension

Yes

17

4.18

No

390

95.82

Gestational Diabetes Mellitus

Yes

5

1.23

No

402

98.77

${ }^{*}$ Neonatal Intensive Care Unit, ${ }^{\dagger}$ Jigme Dorji Wangchuck National Referral Hospital

\section{DISCUSSION}

\section{Neonatal characteristics}

The majority of newborns (78\%) in our study were less than 7 days of age at the time of diagnosis of pathological jaundice. Our study finding was consistent to the observation made by a similar study carried out in Ethiopia showing $90 \%$ of neonates developed jaundice within their first week of life ${ }^{5}$. This may be because, physiologically, neonatal jaundice peaks between three to seven days of age.

The current study found that more than half of the babies with pathological jaundice were males $(60.44 \%)$. Similar findings were also reported in India ${ }^{6}$. In our study, huge percentage of neonates were term and had normal birth weight. Most of the neonates enrolled in our study were admitted in the nursery unit (phototherapy unit) primarily for the treatment of jaundice which includes mostly term and healthy neonates. The remaining small percentage were from the NICU which principally manages pre term babies. This corroborates the findings made in India and Nigeria reporting 81 to $92 \%$ and $83.12 \%$ respectively of term neonates to have pathological jaundice ${ }^{7,8}$.

$\mathrm{ABO}$ incompatibility was found in $22.11 \%$ of neonates but none had $\mathrm{Rh}$ incompatibility in this study. A retrospective study conducted in the central regional referral hospital, Bhutan, in 2018, found that $\mathrm{O}$ and $\mathrm{A}$ blood group were more common blood groups 8 . In the same study only $0.15 \%$ of them were found to be Rhesus $\mathrm{D}$ negative. Thus, there is a higher chance of $\mathrm{ABO}$ incompatibility between the mothers and their newborns in our setting. We did not find any case of Rh incompatibility in our study. The higher prevalence of $\mathrm{Rh}$ incompatibility in different studies could be due to the geographical distribution of $\mathrm{ABO}$ and $\mathrm{Rh}$ grouping.

Twenty-four neonates $(5.90 \%)$ with pathological jaundice had sepsis. As most of our admissions were from the nursery unit where most of the neonates were term and healthy, the number with co-existing sepsis was low. Other studies showed similar findings as well $1^{2,7,9}$.

\section{Maternal characteristics}

A significant association was found between primiparous mothers and excessive weight loss. Excessive weight loss in our neonates born to primiparous mothers could have caused more breastfeeding jaundice or exaggerated physiological jaundice due to increased enterohepatic circulation. Neonates of primipaorus mothers with excessive weight loss could be due to in experienced or little knowledge on good positioning and attachment on breast feeding or could be due to unawareness on feeding adequacy. Aiswarya et al found that $72.72 \%$ mothers were primiparous in India ${ }^{10}$. Similarly, a study by Scrafford et al found significant association between primiparity and neonatal jaundice ${ }^{11}$.

Large number $(77.88 \%)$ of mothers in our study were between 21 and 34 years of age. The higher proportions could be because most of these mothers were in the most reproductive age period between 20 and 30 years. This could also be due to the good family planning interventions inculcated in our health system.

Our study showed an association between primiparous mothers and excessive weight loss in neonates compared with multiparous mothers which was highly significant ( $p$-value of 0.016). A systematic review and meta-analysis by Seyedi et al found that the use of oxytocin increased the serum bilirubin level on the $2^{\text {nd }}$ day of life 9 . Brits et al found that the use of oxytocin was not associated with a higher incidence of neonatal jaundice ${ }^{12}$. This was because majority of the babies included in the study were born via Caesarean section for various indications. However, statistically significant number of neonates developed 
pathological jaundice after inducing/augmenting labor than those born spontaneously in another study ${ }^{13}$.

In our study we observed high prevalence $(63.66 \%)$ of pathological jaundice among neonates admitted at the national referral hospital. This may be because of the large number of deliveries being conducted at the national referral hospital as it caters services to huge portion of Bhutanese population. A similar prevalence $(67 \%)$ of pathological jaundice was observed in a study done in Kerala10. On the contrary two other studies found out lower prevalence (19\% and $22 \%$ ) of pathological jaundice ${ }^{2,14}$. This disparity in findings could be due to difference in the study center where they have included exclusively neonates from the Critical care unit, whereas we included neonates from both nursery unit and NICU. It could also be due to the difference in size of the neonatal population used for the calculation of prevalence.

\section{CONCLUSIONS}

The most common clinical profile of neonates with pathological jaundice observed in our study were $\mathrm{ABO}$ incompatibility, primiparous mothers with feeding issues and neonates less than one week of age. Significant association was found between primiparity and excessive weight loss. The prevalence of pathological jaundice was found to be $63.66 \%$ in our setting.

\section{RECOMMENDATIONS}

The primary health workers involved with mothers and neonates should be made aware of the development of pathological jaundice in neonates and their clinical features leading to severity. Hence during the immediate and subsequent post-natal visits, neonates particularly born to primiparous mothers with feeding issues and excessive weight loss, $\mathrm{O}$ positive mothers with possible ABO incompatibility and neonates less than one week of age should be assessed for jaundice.

A larger, well-designed study could determine associated risk factors and the burden of pathological neonatal jaundice in Bhutan.

\section{ACKNOWLEDGEMENTS}

The authors would like to thank the faculties, staff and my colleagues in the Department of Pediatrics and Faculty of Postgraduate Medicine for their generous guidance and support in conducting the research. We would also like to thank the parents who consented to enroll their neonates in our study.

\section{REFERENCES}

1. Cloherty JP. Manual of neonatal care [Internet]. 7th ed. Cloherty JP, Eichenwald EC, Hansen AR, Stark AR, editors. Early Human Development. Lippincott Williams \& Wilkins, a Wolters Kluwer Business; 2012. [Full Text]

2. Ramsesh A, Ashok D. AIIMS Protocol Neonatology 2019. 2nd ed. Delhi, India: Noble Vision Medical Books Publishers; 2019. [Full Text]

3. Slusher TM, Zamora TG, Appiah D, Stanke JU, Strand MA, Lee BW, et al. Burden of severe neonatal jaundice : a systematic review and meta-analysis. BMJ Pediatr [Internet]. 2017;1(1):1-10. [Full Text | DOI]

4. Olusanya BO, Kaplan M, Hansen TWR. Neonatal hyperbilirubinaemia: a global perspective. Lancet Child Adolesc Health 2018 Aug;2(8):610-620. [PubMed | Full Text $\mid$ DOI]

5. Onyearugha CN, Onyire BN, Ugboma HAA. Neonatal jaundice: Prevalence and associated factors as seen in Federal Medical Centre Abakaliki, Southeast Nigeria. J Clin Med Res 2011;3(3):40-5. [Full Text]

6. Sahoo M, Arigela V, Pramitha L, Sudarsini P, Ku R. Study of neonatal jaundice in a tertiary care centre of South India. Int J Pediatr Res 2016;3(8):585-8. [Full Text]

7. Shetty A, Kumar B. A study of neonatal hyperbilirubinemia in a tertiary care hospital. Int J Med Sci Public Heal 2014;3(10):1289-92. [Full Text | DOI]

8. Chhetri V. Frequency Distribution of $\mathrm{ABO}$ and Rh blood group among Blood Donors at Centre Regional Referral Hospital, Gelephu: A Retrospective Study. J Med Sci Clin Res 2018;6(4):939-42. [Full Text | DOI]

9. Seyedi R, Mirghafourvand M, Tabrizi SO. The effect of the use of oxytocin in labor on neonatal jaundice: A systematic review and meta-analysis. Int J Pediatr 2017;5(12):6541-53. [Full Text | DOI]

10. Aiswarya AT, Sajeeth CI, T AA, Sajeeth CI. The Incidence, Risk Factors and Management of Neonatal Jaundice in a Government Hospital, Palakkad District, Kerela. Int J Heal Sci Res 2016;6(June):123-9. [Full Text]

11. Scrafford CG, Mullany LC, Katz J, Khatry SK, Leclerq SC, Darmstadt GL, et al. Incidence of and risk factors for neonatal jaundice among newborns in southern Nepal. Trop Med Int Heal 2013 Nov;18(11):1317-28. [PubMed | Full Text $\mid$ DOI]

12. Brits H, Adendorff J, Huisamen D, Beukes D, Botha K, Herbst $\mathrm{H}$, et al. The prevalence of neonatal jaundice and risk factors in healthy term neonates at National District Hospital in Bloemfontein. African J Prim Heal Care Fam Med. 2018;1-6. [PubMed | Full Text | DOI] 
13. Mesic I, Milas V, Medimurec M, Rimar Z. Unconjugated pathological jaundice in newborns. Eur PMC 2014;173-8. [PubMed | Full Text]
14. Sharma S. Neonatal Hyperbilirubinemia: Hospital Based Study in Western Region, Nepal. Janapriya J Interdiscip Stud. 2016 Jul;5(December):75-82. [Full Text | DOI]

\section{AUTHORS CONTRIBUTION}

Following authors have made substantial contributions to the manuscript as under:

KD: Concept, design, data collection and analysis, manuscript writing and review.

TL: Concept, design, manuscript writing and review

MLM: Concept, design, manuscript writing and review

P: Concept, design, manuscript writing and review

Author agree to be accountable for all respects of the work in ensuring that questions related to the accuracy and integrity of any part of the work are appropriately investigated and resolved.

\section{CONFLICT OF INTEREST}

None

\section{GRANT SUPPORT AND FINANCIAL DISCLOSURE}

Ministry of Health, Royal Government of Bhutan 